\title{
Integrated Domain in Support of Game-Based Learning in School Education
}

\author{
Stanimir Stoyanov ${ }^{1,2}$, Todorka Glushkova ${ }^{1}$, Veneta Tabakova-Komsalova ${ }^{1,2, *}$, Vanya Ivanova ${ }^{1}$, Asya \\ Stoyanova-Doycheva ${ }^{1,2}$, Alexandar Petrov ${ }^{1}$
}

${ }^{1}$ Faculty of Mathematics and Informatics, University of Plovdiv “Paisii Hilendarski”, Plovdiv, Bulgaria.

${ }^{2}$ Institute of Information and Communication Technologies, BAS, Sofia, Bulgaria.

\begin{abstract}
How to cite this paper: Stanimir Stoyanov, Todorka Glushkova, Veneta Tabakova-Komsalova, Vanya Ivanova, Asya Stoyanova-Doycheva, Alexandar Petrov. (2021). Integrated Domain in Support of Game-Based Learning in School Education. The Educational Review, USA, 5(11), 434-446.

DOI: 10.26855/er.2021.11.004

Received: October 9, 2021

Accepted: October 31, 2021

Published: November 30, 2021

Corresponding author: Veneta Tabakova-Komsalova, Faculty of Mathematics and Informatics, University of Plovdiv "Paisii Hilendarski”, Plovdiv, Bulgaria; Institute of Information and Communication Technologies, BAS, Sofia, Bulgaria.

Email: v.komsalova@uni-plovdiv.bg
\end{abstract}

\begin{abstract}
Educators are looking for more and more interesting and attractive forms of e-learning, especially in times like the current crisis related to the COVID-19 pandemic. Most universities and secondary schools use e-learning environments and develop teaching materials. It would be interesting to share them in a single so-called integrated domain. This article presents a platform known as ViTOS supporting game-based learning in an integrated educational domain that includes university education and training in STEM centers of the secondary school. The front-end and the back-end components of the platform are also described. Furthermore, a personal assistant conducting the running of the games is presented as well. The use of the platform is demonstrated by two examples from two different domains-smart city and smart agriculture. The games are conducted for real education in a school STEM center and in a university e-learning environment. Ideas for future improvements are also presented.
\end{abstract}

\section{Keywords}

Educational Platform, Game-Oriented Education, Integrated Domains, Personal Assistants, Smart City, Smart Agriculture

\section{Introduction}

Educators are looking for more and more interesting and attractive forms of e-learning, especially in times like the current crisis related to the COVID-19 pandemic. For years, our team has been working to create a reference software architecture called ViPS (Virtual-Physical Space) (Stoyanov, Glushkova, Doychev, Stoyanova-Doycheva, \& Ivanova, 2019). The construction of ViPS is encouraged by the concept of CPSS (Cyber-Physical-Social System), which focuses on the integration of the virtual, physical, and social worlds (Wang, 2010). Over the years, ViPS has been adapted to develop applications for e-learning, cultural tourism, and smart cities (Glushkova, Stoyanova-Doycheva, Ivanova, Stoyanov, \& Doychev, 2019; Stoyanov, Orozova, \& Popchev, 2018).

In 2020, the team was included in the National Scientific Program (NSP) "Smart Agriculture" to adapt ViPS for intelligent agriculture. One of the accompanying goals of the program is to involve children from an early age in ecological thinking, healthy eating, and technological discipline. Another objective is to create habits for evaluation and active involvement in various initiatives useful for a healthy and safe life such as safe movement, clean nature, and healthy food. In our opinion, for the successful application and implementation of such learning objectives in high school, it is necessary to use and combine traditional and modern forms, methods, and approaches with active 
involvement of elements of the research and problem approach, as well as opportunities for demonstration and laboratory experiment. In this way, students are given the opportunity to find meaning in what they have learned and discover an application in new and unfamiliar situations. For this purpose, a new component called ViSMod (Virtual Space Modeling) has been developed in the adapted version of ViPS, which is focused mainly on modeling scenarios and processes of smart agriculture, smart environment, and smart cities. As part of the NSP, one of the goals of ViSMod is to become the core of an e-learning platform in high school supported by the university. In this distributed infrastructure, ViSMod will act as a back-end party providing learning material in the form of games. As a front-end part, we have developed a separate component to be located in the STEAM center of the respective high school. The working name of the infrastructure is ViTOS (VisMod TO Steam).

The idea to build such a platform arises in connection with the research of the so-called integrated domains. As mentioned, ViPS is adapted for different application areas. The space includes standard components (usually, these are formal systems for modeling the spatial, temporal, and event aspects of physical objects) that adapt to domain-specific data structures (the application area). Furthermore, in certain cases, it is necessary to develop new, strictly domain-specific components. These new components are stored in specialized libraries, which become an integral part of ViPS. Thus, with each adaptation, the reference architecture is expanded and enriched with new components. At the same time, in some instances, it would be appropriate for ViPS to refer to more than one domain. In this case, the integrated domain consists of two domains - university education and high school education.

In this article, we present the general architecture of an environment for an integrated educational domain. The next section provides a brief overview of current projects in this area. The third section briefly describes the platform. The fourth section demonstrates the usage of the platform by means of two examples. The article ends with a conclusion and future work section.

\section{Related Works}

In Dobrowolski, Hanusz, and Sobczyk (2015) and Borecki, Tolstych, and Pok (2013), it was found that as early as the late 70s of the last century, research was conducted with reference to video games and motivational effects associated with learning as well as the cognitive potential of computer games. Even back then, the results of the study showed that the visual and motor coordination of players was better than that of non-players. The initial study also revealed the importance of electronic games for children who find it difficult to learn basic subjects and skills. Video games help students to identify their shortcomings and try to correct them. The adaptability of video games and the control that players have over them motivate and stimulate learning.

The use of games in education continues to this day and this field is becoming the subject of growing scientific and practical interest. Here, we will briefly present some interesting contemporary developments. Video games are turning into an increasingly powerful tool for engaging the public in climate change, but little is known about how they can play this role. Therefore, the study of more than 150 video games representing climate change is extremely relevant, the results of which are summarized in (Galeote \& Hamari, 2021). In order for the games to be analyzed and statistically compared, a system of 15 dimensions is used. The analysis shows that most games meet the recommended attributes; however, credibility, achievability, meaningfulness, and social features are still rare. The findings reveal that researchers would benefit from broadening their horizons to find neglected opportunities for engagement, communication, and education in the face of climate change. Another current objective of game-based learning is IoT (Internet of Things) applications. Thus, for example in (Bahrini et al., 2020), a game is presented in order to inform users about the challenges related to the security of a smart home. While smart home systems are spreading fast, user privacy and security are key issues. Many users find it difficult to obtain and implement security recommendations to protect against malicious behavior in these systems, which can cause a loss of interest in the topic. Game-based learning can help to increase user motivation in a fun and intuitive way. Two versions of the game with opposite game assumptions (well-intentioned and malicious) have been developed, which are compared in an experiment. The results show high ratings of motivation in both versions of the game to solve the security problems of a smart home. A project is presented in Amogh Joshi (2020), aimed at making learning activities optional, but at the same time, maintaining the commitment of players to the educational content. To test this idea, four variants of the game were developed by integrating educational material into different game mechanisms. A specific mechanism has been chosen that motivates players to voluntarily get involved in solving computer puzzles. Data structures and algorithms are fundamental concepts studied in the first semesters of any bachelor's degree program in informatics. Many students find these concepts a serious challenge, partly because of their abstract nature, but also because of problems with low motivation. In (Simon Su et al., 2021), these and other common difficulties faced by teachers are addressed, presenting a new solution that combines a game-based learning approach with the use of engaging visualization of algorithms. Game-based learning and algorithm 
visualization are often used in existing educational tools, but they are rarely combined. In this game, abstract concepts are presented as objects and animations in a 2.5D world. Feedback from an initial pilot study suggests that students view the game as a pleasant and effective tool for learning data structures and algorithms concepts. Evidence has also been found that visualizations help students to find effective solutions to problems. In Juho Kahila, et al. (2020), a game for primary school is presented, which mixes physical and virtual elements and engages students to explore a room. The sound elements with speech, songs, and background sound landscape, combined with intelligent lighting, enhance the "immersion" in the image created by the game. The game can be packed in two small suitcases and placed anywhere. With the ubiquity of artificial intelligence in everyday applications such as smartphones and social media, children need skills and digital literacy to navigate the digital world and to critically view and reflect on the social and ethical implications of using intelligent systems. To address this growing need, especially for younger students, the LearnML project was presented in (Voulgari et al., 2021), which aims to develop a framework and game-based educational materials to promote literacy in artificial intelligence among students from the primary and secondary education.

\section{General Architecture of ViTOS}

The ViTOS platform consists of two basic modules:

- $\quad$ Back-End Module - the ViSMod environment will operate as such;

- Front-End Module — the STEAM school centers will operate as such.

\subsection{ViSMod as the Back-End Module of ViTOS}

VisMod is an environment for modeling processes, scenarios, technical means, and systems that can be elements of future smart infrastructures, such as smart agriculture or a smart city. An accompanying function of the environment is to be used for various forms of e-learning (especially game-based learning), mainly in the school subjects of artificial intelligence, biology, ecology, and agriculture. ViSMod is being developed as an expert system consisting of four basic components—Knowledge Base, Simulator, Explanation, and User Interface (Figure 1).

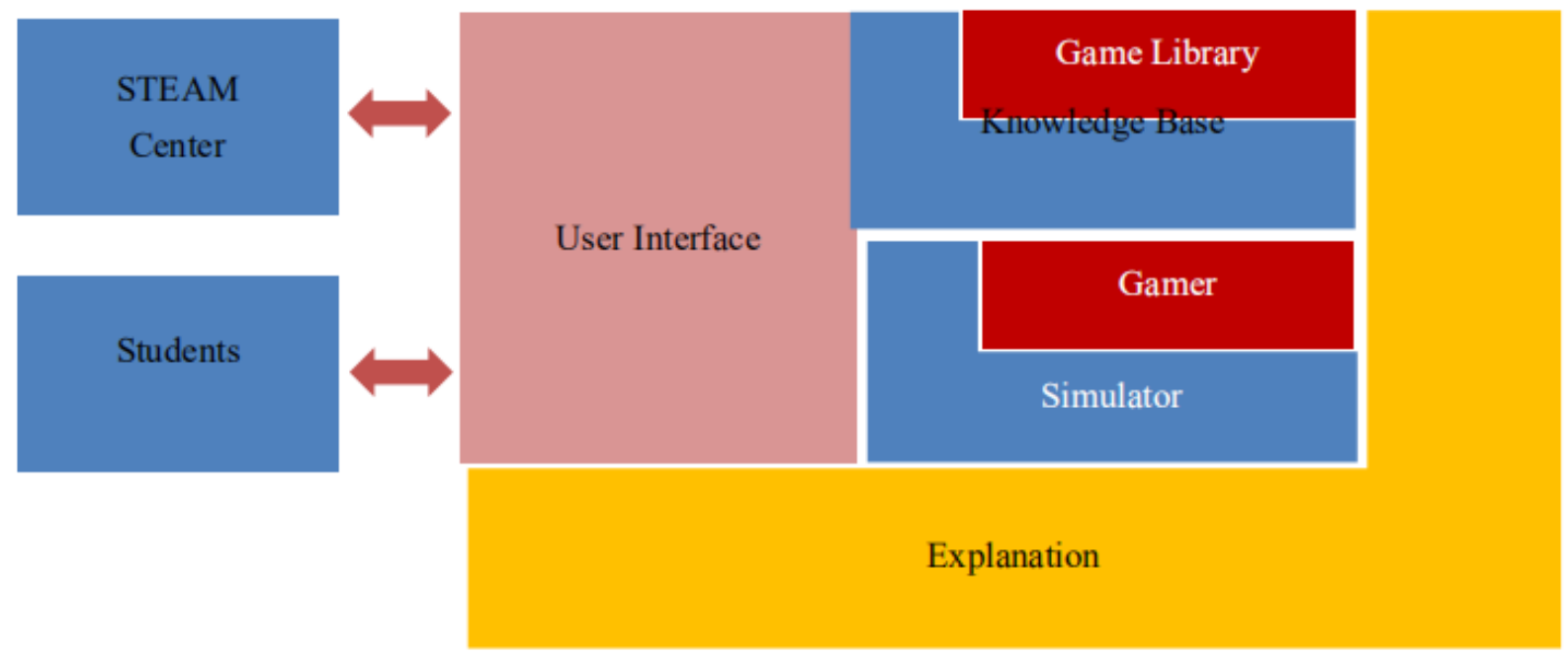

Figure 1. ViSMod Architecture.

Knowledge Base. The Knowledge Base is a distributed repository for storing domain-specific knowledge. In general, the repository saves knowledge about the natural and the artificial worlds. Currently, the Knowledge Base consists of the following five bases for the natural world - soil, air (climate), water, plants, and animals, as well as a base for the artificial world (Figure 2). One of the components of the Knowledge Base is the Game Library, which stores games. The Game Library is serviced by a specialized editor, with the help of which libraries with domain-specific building blocks can be created. The building blocks are used to prepare specific game scenarios (as shown in the example) in the user interface. Such building blocks for a smart city can be buildings, streets, traffic lights, cars. Examples of building blocks for agriculture can be crops, farm animals, water pumps, valves, hoses. There are also shared building blocks for both domains (especially from the artificial world), such as sensor and communication networks, drones, robots.

In the Game Library, a game is specified in the following three parts: 
- The logic (and limitations) of the game, presented as rules;

- Meta-data for the personal assistant (discussed in more detail below);

- Correspondence to the building blocks included in the specific game scenario.

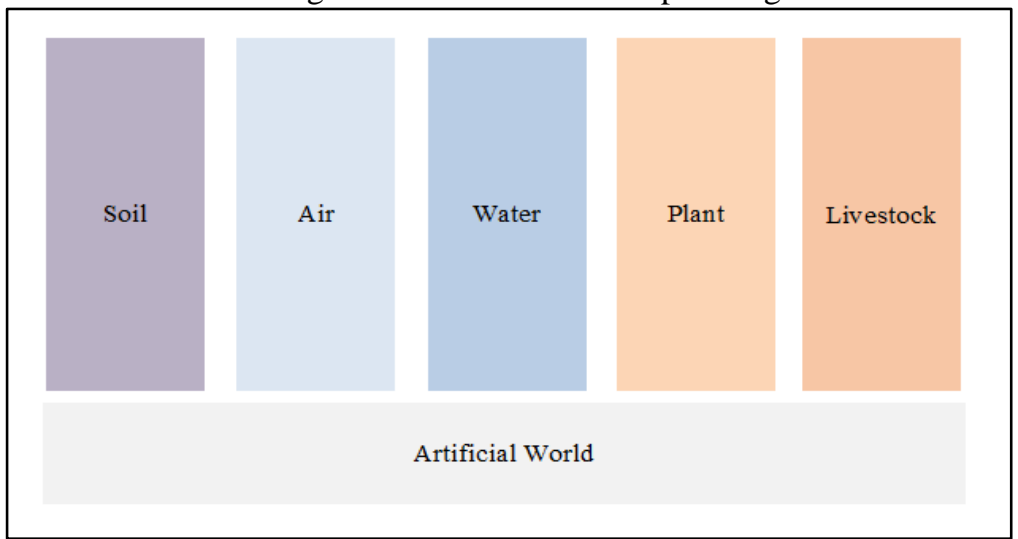

Figure 2. Structure of the Knowledge Base.

Simulator. The simulator is a ViSMod output machine that can interpret the models and knowledge stored in the library, as well as external knowledge. Similar to the Knowledge Base, the simulator has a built-in module called Gamer. The Gamer is a type of interpreter with the ability to activate and control a selected game. The Gamer core is a personal assistant, which is discussed in Section 3.3.

Explanation. When managing a game, the Gamer (the personal assistant in particular) keeps a protocol for the course of the game, the dialogue conducted with the player, and mistakes made by him/her. This protocol is recorded in the Explanation. Subsequently, the teacher has access to this data and he/she can analyze it accordingly.

User Interface. It is used to interact with ViSMod users. University users can have direct access to the system. High school users access ViSMod through the Front-End module of the platform described in the next section.

\subsection{Front-End Module}

The front-end module is located in the STEAM center of the school. The module is developed as a game-based learning environment. The architecture of the module includes the following four layers (Figure 3): Data Base Layer, Web Services Layer, an interface to external systems, and a user interface.

- Data Base Layer. It stores data about games, users, and their statistics. The users are actually individual mobile devices. Thus, no personal data is collected and no registration is required, as this is inappropriate for a child-friendly application.

- Web Services Layer. It contains the business logic of the application with four main modules-Administrative (Admin), Personal Assistant, an Application Programming Interface of the server (Backend API), and a Notifications Manager (Push Notifications Manager). The Admin contains the user interface for administrative tasks performed by super users and administrators. Examples include uploading game content, granting and revoking permissions, and others. They also act as curators of player statistics. The Backend API is a standard REST API used to communicate between the client side and the server. The only thing that should be noted here is that the player's statistics are not recorded directly in the database, but are transmitted to the intelligent personal assistant so that it can analyze and evaluate them beforehand. The Push Notifications Manager is used by the personal assistant of the server and the API of the administrator. It is responsible for sending personalized messages to the mobile client via Google Cloud Messaging and the Apple Push Notifications Server.

- Interface to External Systems Layer. One of the external systems, used in the architecture, is the cloud of the University of Plovdiv, through which the connection with the back-end module is made and, accordingly, it becomes a Content Delivery Network (CDN). The games in the STEAM center are delivered through this interface from the game library, which is stored in ViSMod. The second external system is Google Cloud.

- User interface. It includes two client applications—a mobile client for players (students) and a web client for administrators (teachers). Students can conduct the game through the interface, which operates as a personal assistant, presented below. Teachers can administer the games and view player statistics. The user interface provides an API for an administrator web client. Its main purpose is to provide management mechanisms for data and metadata. 
This module is responsible for collecting data displayed to the mobile customer and for enforcing compliance with the terms of use. Within the administration module, there is also an integrated promotion module, which notifies end users of new games added to the game library.

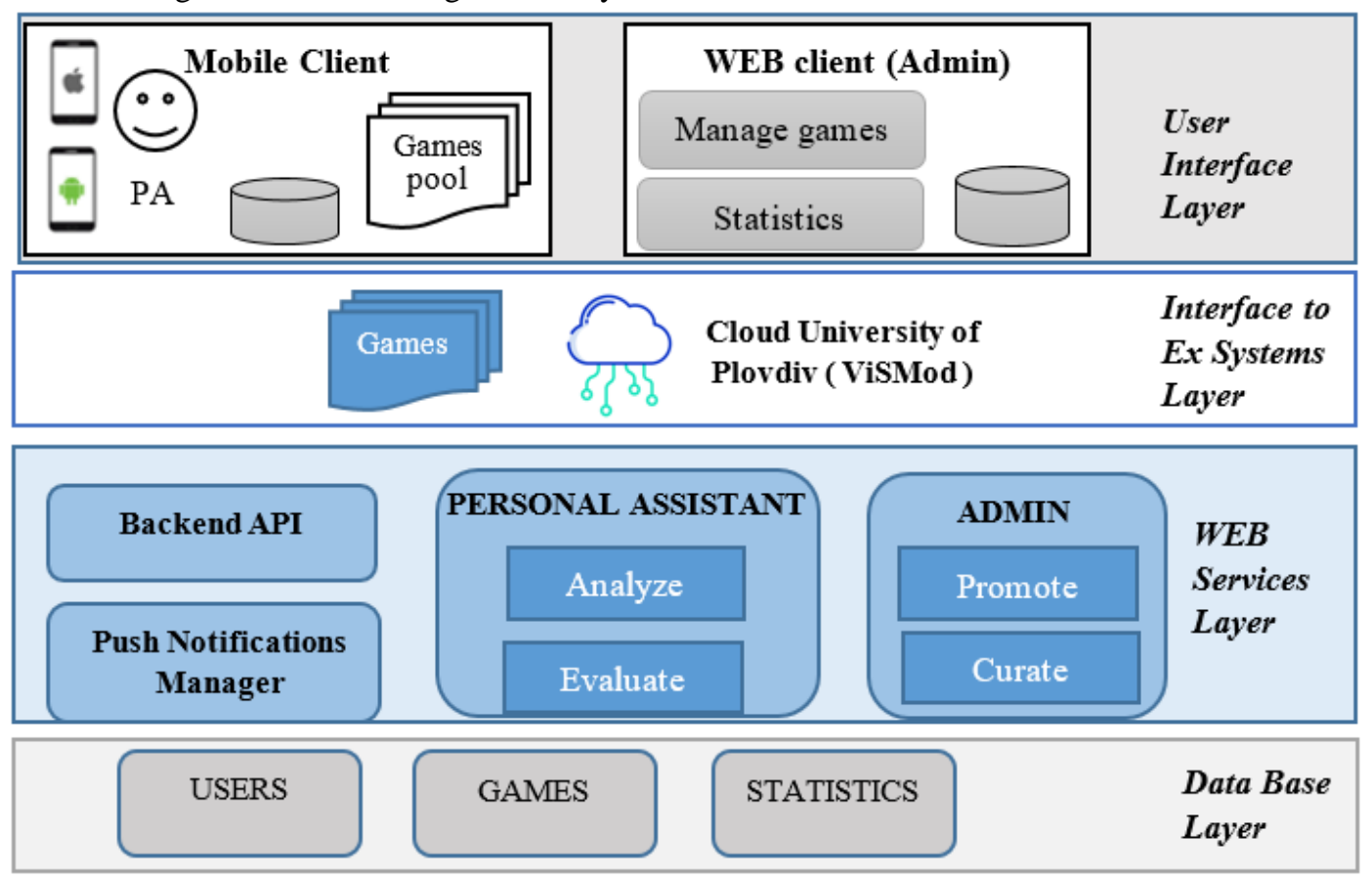

Figure 3. Front-end, located in the STEAM center.

\subsection{Personal Assistant}

The basic component of the Gamer is a Personal Assistant (PA), implemented as an intelligent rational agent (Wooldridge, 2009). As such, the PA is autonomous, reactive, proactive, and social. The task of the PA is to support the work of users (children, students, teachers) with the ViTOS platform. It can promote, guide, publicize, and offer games. The personal assistant, being proactive, monitors the player's progress and, if necessary, activates a dialogue with him/her to provide assistance or, in certain situations, it takes the initiative to conduct the game. Another task of the PA is to record the course of the game and collect a variety of information in the environment. This information is recorded in the explanatory component and can be subsequently analyzed by teachers. In fact, the personal assistant is a multi-agent system, the architecture of which is transparent to users. The personal assistant consists of two parts; one is located on the server and the other one-in the mobile client of the STEAM center.

- Personal Assistant-server side. The personal assistant—server side is the "brain" of the architecture, as it is implemented as an intelligent software agent and is used to analyze the player statistics and the behavior while using the application. It monitors not only the points scored during the game, but also the time of the player when using or not using the application as well as his/her preferences within the application. Depending on the assessment made by the personal assistant, proactive behavior may be triggered by the notification manager. A simple example of this is the case where the application has been inactive for several days. Then it will send a kind reminder that it is time to play and learn.

- Personal assistant-Client side. The main role of the personal assistant is to guide and encourage players to progress in the educational games offered. It sends messages to users via audio and text. It is actually associated with a woman in the role of a teacher. Currently, we have pre-defined many text and audio messages in Bulgarian. While using the application, messages are triggered by various events. It starts with a greeting, depending on the launch time of the application, so it could be, "Good morning! What do you want to play today?", or "This is a good day to play and learn”, or “Isn't it too late to learn?”, etc. (Figure 4). Arbitrariness is also applied when choosing a greeting, recording the last salutation to the player so that the next one can be different. There are also various messages throughout the application lifecycle. For example, a message encouraging to stop the application will be triggered after a certain period of time. The personal assistant can receive messages from the server and ac- 
tivate proactive behavior. The personal assistant—client side is usually located on the mobile device of the students. The developed application has customization options. For example, notifications can be disabled and rest time can be changed or deactivated; in addition, students can change the theme of the user interface during the application execution and save these settings. According to many studies, children with autism sometimes have atypical color preferences and find certain colors soothing (Petrov, Epitropova, \& Stoyanov, 2020). The information screen has useful data that is displayed not only for children but also for their parents or their teachers. There are instructions on how to use the application. The data collected by the device includes how many times the application has been opened, what the total usage time is, and how long the application has been inactive (in the background). In the future, it is planned to monitor the frequency and duration of use of specific games. This would help the personal assistant to offer different games in case of excessive use of the same one.
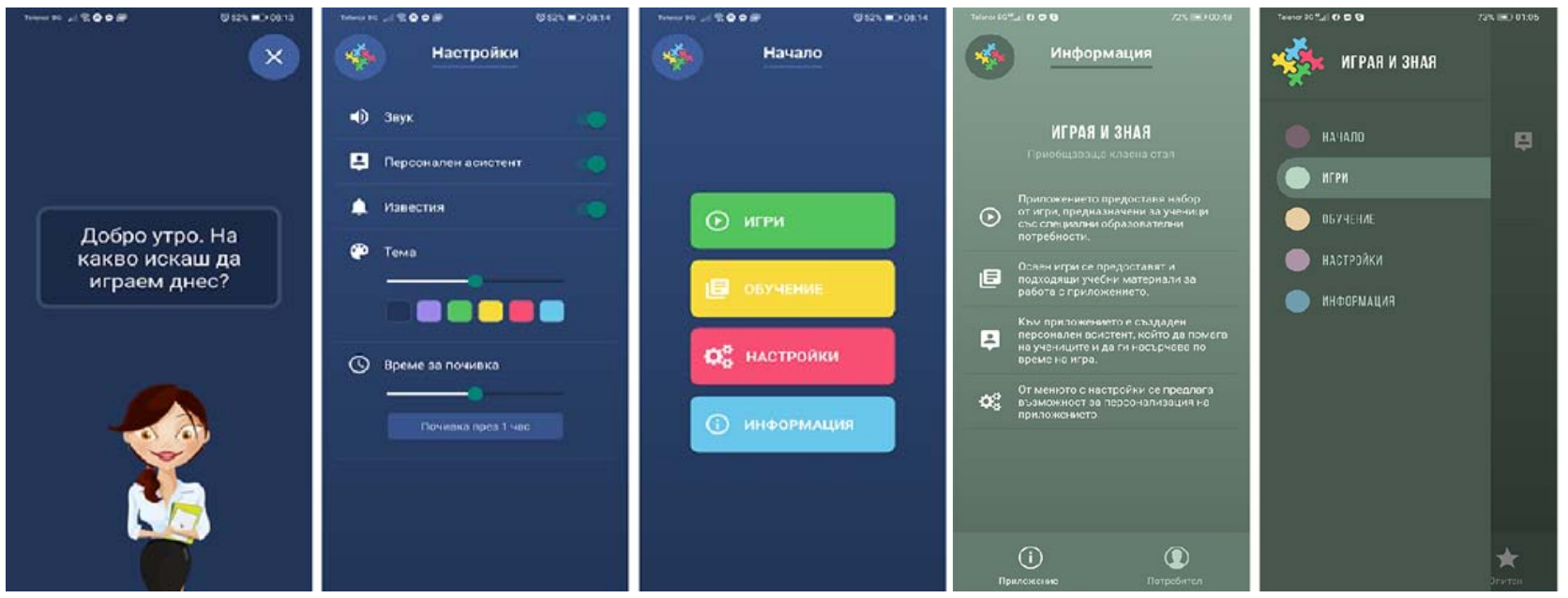

Figure 4. User interface-mobile application.

\section{Educational Games}

In recent years, due to the circumstances surrounding the COVID-19 pandemic, the infrastructure proposed in the article has proved to be a suitable aid to support high school learning. Interdisciplinary lessons are organized with small groups of students in the STEM center, including subjects from the natural sciences, mathematics, technology, and other scientific fields. We will demonstrate the use of the infrastructure with two sample lessons - the first one is from a smart city and the second-from smart agriculture.

Game-based learning is used in the suggested lessons. This training seeks to balance the school subjects in the purpose of the game and the player's ability to apply what is learned in the real world. In the STEM center, through collaboration between teachers and learners, depth and perspective are added to the experience gained from the game. The benefits that game-based learning provides are well known (Nistor \& Iacob, 2018). In this context, they increase motivation and engagement, provide a safe model of the world that is close to reality, improve learning and memory, stimulate creativity and enhancement of soft skills, encourage self-discipline and control, provide monitoring of the training process, etc.

The smart city course provides an opportunity for training in road safety (RS), which is an integral part of the overall education, training, and socialization of students in the school education system. This is important for the formation of certain practical skills in students as participants in the road traffic, especially in the context of the restrictions imposed by COVID-19.

The new European Policy Framework "Next Steps towards 'Vision Zero"” sets the focus of the Union GDP policies for the following decade 2021-2030. Together with the adopted long-term vision of zero deaths by 2050, the EU has set new milestones to reduce fatalities and serious injuries by $50 \%$ by 2030 . Road safety education and training are essential and should be seen and implemented as a comprehensive process of "lifelong learning". GDP training is considered an integral part of road safety, as it helps to correct the behavior of road users. A common indicator for determining the effectiveness of road safety measures is the reduction of deaths and accidents.

Road safety (RS) training is conducted in all European countries at different levels of secondary education. In Bulgaria, RS education is mandatory in all types of kindergartens, schools, and centers for personal development. GDP train- 
ing is a mandatory part of the school curriculum. The responsible people for its implementation are class tutors or teachers of specialized subjects. It is also conducted by public road safety organizations, NGOs, and parents. The planned number of school periods for GDP training is extremely insufficient. According to the specialized agencies, the effects of training in this area are difficult to determine and their measurement is not possible only by the amount of road accidents as a major marker. Research at the national level for Bulgaria shows that the active conduct of such training has a positive impact on the attitude and behavior of students and citizens provided that the training program is well structured. For this reason, we hope that through games in STEM centers in the free time of students, one of the main goals of the training will be achieved - demonstration of responsibility and self-control as road users.

To achieve the main objectives of the training, according to the Bulgarian Sectoral Strategy for Road Safety (2021-2030) of the Ministry of Education and Science from December 2020, it is necessary to (Bulgarian sectoral strategy for road safety (2021-2030):

- Form initial ideas about the road environment; build and develop protective mechanisms against the dangers of the road environment.

- Develop sensory perceptions (sight, hearing, perceptions of time and space) for safe behavior on the road.

- Develop the concentration of attention and memory; form skills for safe behavior and culture on the road.

- Acquire knowledge and skills for safe movement with and without a vehicle, crossing at a traffic light and a pedestrian path, use of public transport, etc.

The realization of these goals using the proposed game-based approach is presented by the example below.

\subsection{Educational game that helps learn the rules of the road}

A specialized visual editor has been developed for the preparation of game scenarios. Players (students) or administrators (teachers) or both can develop a scenario suitable for the educational purpose (e.g. going safely from home to school). The editor allows students to construct the virtual world themselves, which is especially useful for the development of their spatial and sensory perceptions. The editor uses a repository with building thematic blocks, from which models and scenarios of the city and the road situation can be developed that differ in complexity. There is a panel for placing the objects on a selected surface, with the possibility to rotate the graphic resource, delete the object, ask a question on the generated route, or define an entry point for the player (Figure 5). The visual editor greatly simplifies the administrator by providing an interface for easy replacement of objects (buildings, road signs, traffic lights, etc.). Subsequently, objects (generally buildings and trees) are placed at random for each impassable cell, and road signs and traffic lights are added to the road markings. The various built-in control points, which serve to guide the personal assistants in the environment, remain transparent for users.

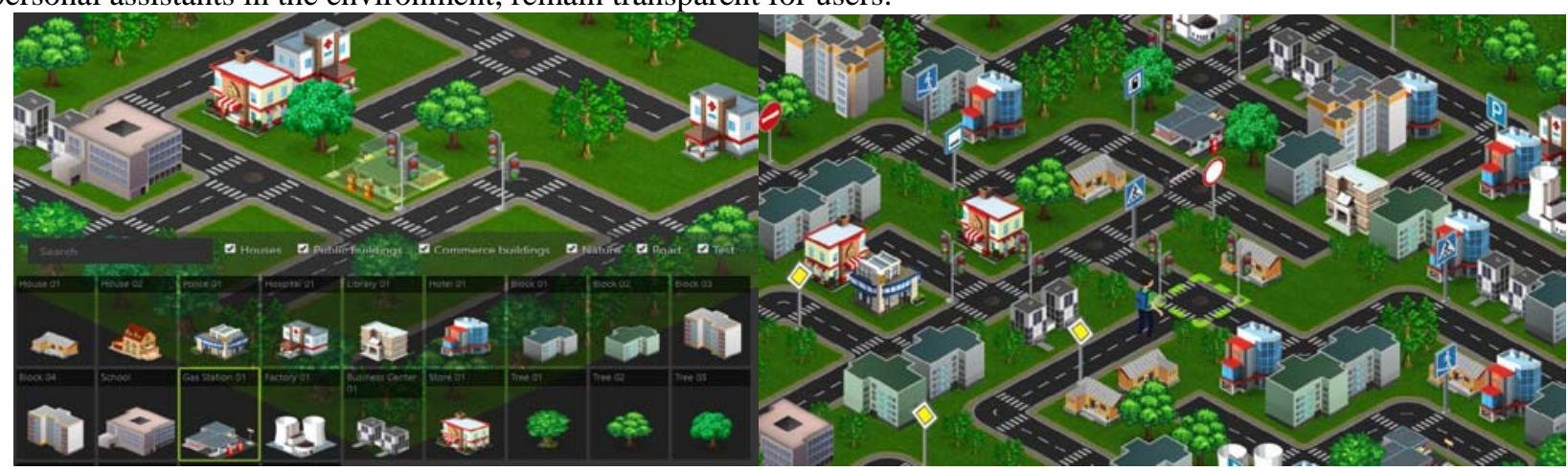

Figure 5. A visual editor for generating a virtual city model and a game scenario.

The editor can be adapted for a script from another application area, e.g. smart agriculture. For the adaptation, it is necessary to integrate a repository with other thematic building blocks suitable for the new school subject.

The administrative part aims to meet the needs of users with the role of a teacher. In this environment, teachers have the opportunity to add new students, create exams, or edit existing ones and check the exam results of the players (Figure 6a). The results of each game are presented to the teacher in tabular form. Figure 6b provides the result of a game (test) of a student, in which all the indicated answers during the game are visually presented to the teacher. 


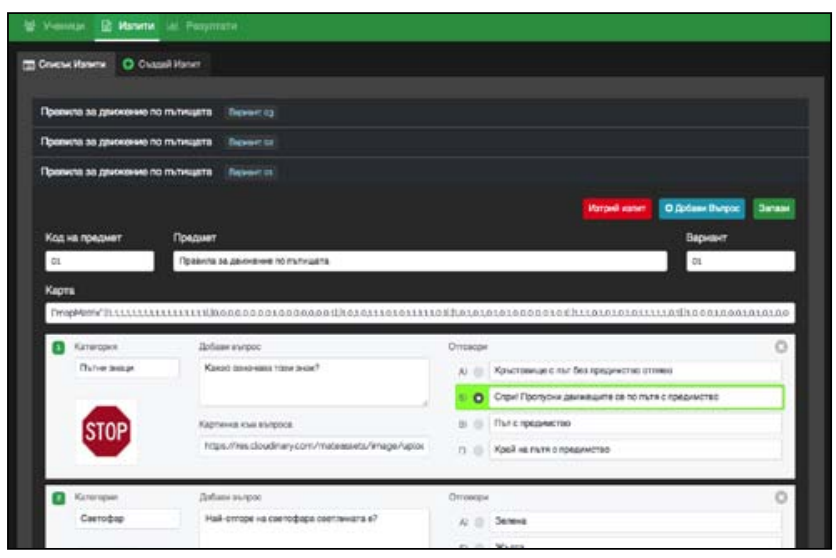

(a)

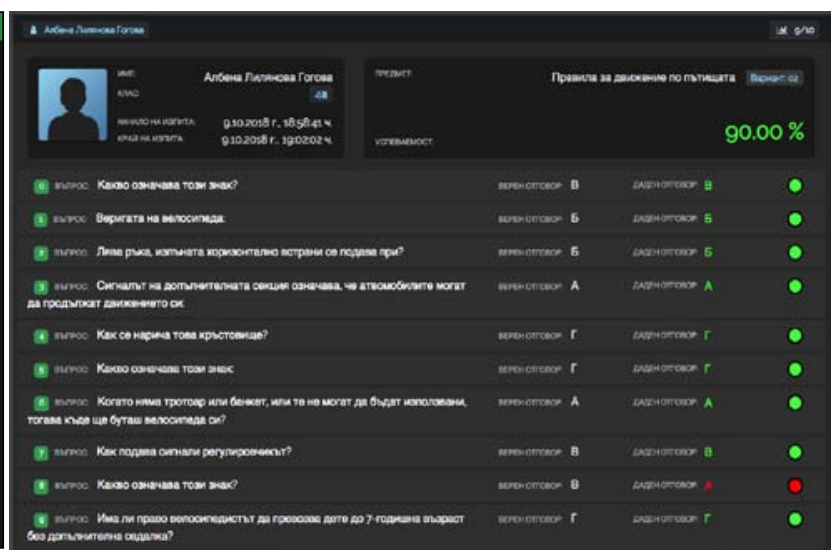

(b)

Figure 6. Administrative part-(a) creating and editing a game and (b) reviewing the result of a game.

The game environment for students provides two separate modules:

- A web-based game environment, and

- A Mobile gaming environment.

The web-based game environment is built using the Backbone.js framework and it is distributed as a browser application. The student's game proceeds as follows: the student chooses his/her profile, then selects a game and follows a turn-based movement in the virtual world provided to him/her (Figure 7). On each move, the student moves forward, being asked a question from the pre-defined categories, corresponding to the virtual traffic situation in which he/she is. At the end of the exam, the player receives a message about the successful completion of the test and is presented with the result of his/her game. In case of a wrong answer (marked in red), the correct one is indicated (in green). The goal is for students to have an immediate opportunity to learn the correct answer.
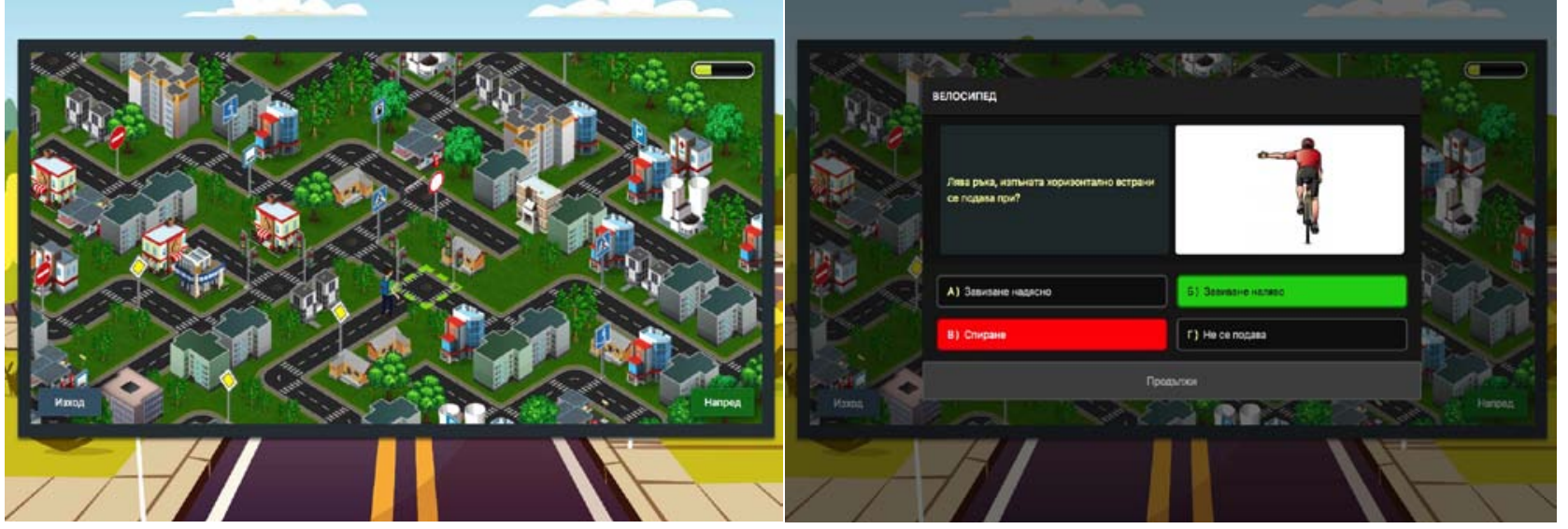

Figure 7. WEB-based game environment.

The mobile game environment is also designed for the user role of a student. It was created with the React Native framework, allowing the export of the mobile application for the two main mobile platforms currently existing-Android and iOS. In order to easily customize and distribute the application, the mobile application has been added to the overall software architecture. The prototype version of the game has been added to Google Play for easy installation and use.

The data obtained from the application of the game platform in school practice shows high commitment and interest. The success rate is very high due to the competitive nature of the training and the interactivity of the virtual reality used. The statistics related to the mistakes made are more interesting. Although students prefer games connected with pedestrian and bicycle questions, their errors in this area amount to almost $50 \%$, while on more difficult topics the errors are relatively fewer. This is probably due to the increased level of concentration during the game and the use of virtual reality, in which it is easier for students to master the otherwise abstract topics. In addition, it is noticed that in 
each subsequent game the students make fewer and fewer mistakes (Figure 8).

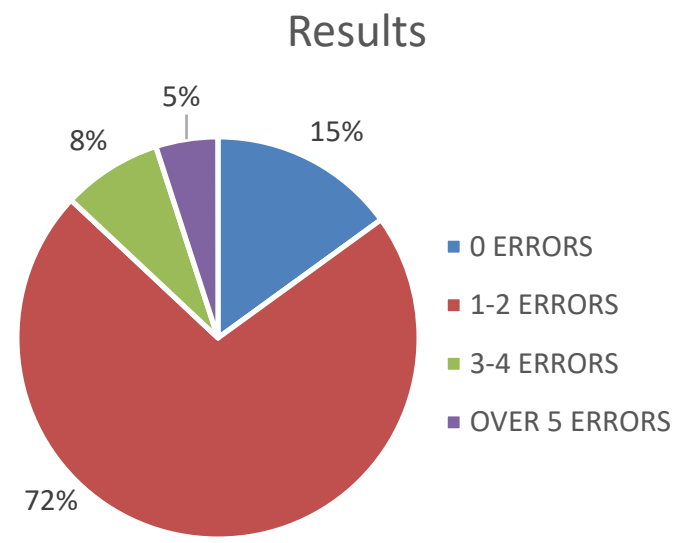

\section{Errors by individual categories}

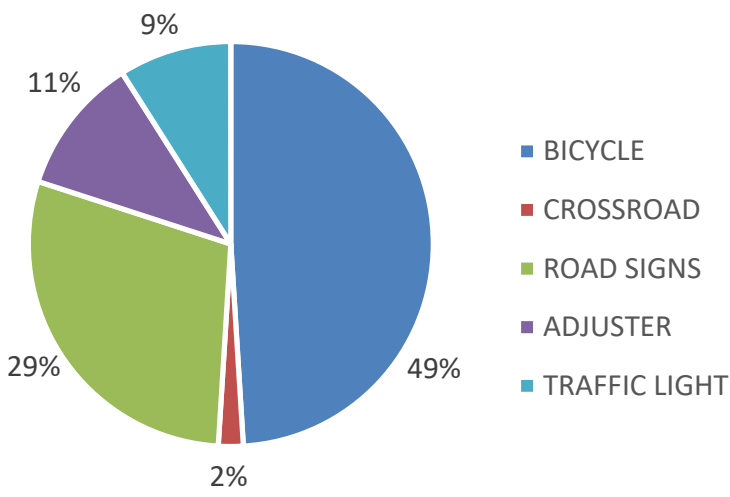

Figure 8. Results from the experimental training with the game environment in STEAM centers.

The considered multi-agent environment for game-based road safety training integrates knowledge about civic education, physics, transport, mathematics, etc., into a common domain. It can be easily and conveniently applied to teaching students in other fields such as biology, ecology, geography, history, and so on. It is necessary to model another virtual environment and replace the database with questions from other application areas.

\subsection{Educational game that helps to learn crops}

The second scenario for an interdisciplinary lesson is focused on smart agriculture and includes school subjects from the field of natural sciences, mathematics, computer science, technical sciences, and others. The curricula in science, ecology, and technology in high school are aimed at developing skills to support sustainable development and encourage a healthy lifestyle and doing sports, and more specifically, understanding the relationship between man and the environment, interpreting specific behavior as a consequence for personal and public health, the use of health knowledge, decision-making, assessment of health risks in a specific situation, and support of activities intended for protection of the environment and personal health (Republic of Bulgaria, Ministry of Education and Science, Educational programs). The main emphasis in the technical sciences is on the formation of the part of the technological competence that is related to the independent development of projects, in which accessible software products and digital means for communication and control are applied. An attitude towards a healthy and safe way of life and work is formed, as well as towards observance of ecological norms of behavior in the environment. Science curricula include school biological knowledge relating to the diversity and classification of organisms on Earth, their role in nature and for humans, relationships in nature, and the conservation of biodiversity, the natural environment, personal and public 
health.

This game scenario is based on the popular "Twenty questions game", which motivates deductive decisions and creativity. The game originated in the United States in the 19th century but its popularity increased dramatically in the 1940s when it began to be widely played on radio quizzes. The most popular version of the game is designed to recognize what object it is (e.g., an animal, a plant, a mineral). In our version, the scenario is called "Recognize the crop". The plot (course) of the script is quite simple-by activating a dialogue with the player, the game tries to guess the crop he/she has in mind. For this purpose, the game uses its base of background (initial) knowledge and the answers of the players.

In the realization of the presented game scenario, we use two approaches. In the first one, which we have tentatively called "The game teaches the student", the student formulates a certain hypothesis in the beginning. In a dialogue with $\mathrm{him} / \mathrm{her}$, the system consistently checks and accepts or rejects a given hypothesis on the basis of its knowledge and the asked and respectively received answers to questions. When a hypothesis is rejected, the inference machine (the Gamer) looks for another possible hypothesis (Figure 9). If, based on its knowledge, the Gamer does not find any possible hypotheses, the game ends with a negative result. In this case, by linking this approach to the idea of game-based learning in agriculture, students can test a hypothesis for a particular type of crop (e.g. vegetables) by answering the questions of the game. In this scenario, the university domain acts as a "teacher" and the school domain as a "learner". Working in the STEAM center (for example, a vegetable garden), the student can formulate a certain hypothesis. $\mathrm{He} / \mathrm{she}$ wants to test whether this hypothesis is true so the student's personal assistant accesses the ViSMod game library and initiates a dialogue between the student and ViSMod.

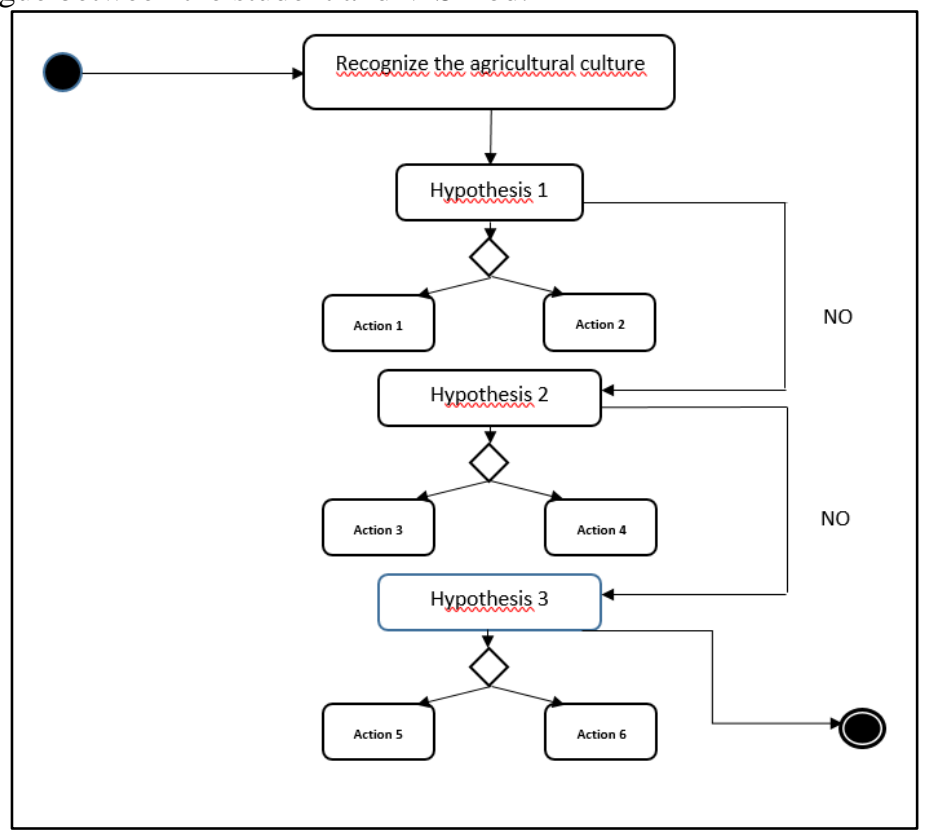

Figure 9. Game control scheme by the Gamer.

In the second approach, which is called "The student learns the game", the roles are reversed-the school domain is a "teacher" and the university domain is a "learner". In this version of the game, students can teach the game by conducting a dialogue with it. In this game scenario, the Gamer uses dynamically changing tree structures. When the game is activated, the Gamer initiates a dialogue to find out what crop the student has in mind. If the student answers negatively to a question, the Gamer wants to expand its knowledge with a new hypothesis. For this purpose, it asks the student questions. Thus, the game tree expands dynamically with the new hypothesis, and the game becomes "more knowledgeable".

We will demonstrate the conduct of the game with a concrete example. Let us suppose that in the knowledge base we have the following knowledge: "The crops of the potato family are typically ethnobotanical, i.e., widely used by humans. They are an important source of food, spices, and medicines. Some of these crops are edible, while others are poisonous. They are rich in alkaloid glucosides, which, in toxicity to humans and animals, can vary from mildly irritating to fatal even in small amounts. The potato family includes the following crops: eggplant, tomato, mandrake, pepper, tobacco, belladonna, tatul, petunia”. When starting the game, the Gamer initiates the following dialogue (the 
student's answers are given in red):

?- go.

Is it ethnobotanical? yes.

It is a tomato? |: no.

What crop are you thinking of?

I: belladonna.

What yes/no question distinguishes it?

I: 'Is it poisonous?'.

For belladonna the answer is: I: yes.

New game? |: yes.

Is it ethnobotanical? |: yes.

Is it poisonous? |: yes.

It is belladonna? |: yes.

New game? |: no.

true.

In the beginning, based on the background knowledge from the Knowledge Base, the Gamer builds the game tree presented in Figure 10a. During the dialogue, in the tomato node, it finds a contradiction between the background knowledge and the player's response. In this case, the Gamer requests additional information and consequently replaces the tomato node with a subtree, as shown in Figure 10b. The next time the game is started, the Gamer uses the new knowledge presented in the game tree, which can be expanded again.

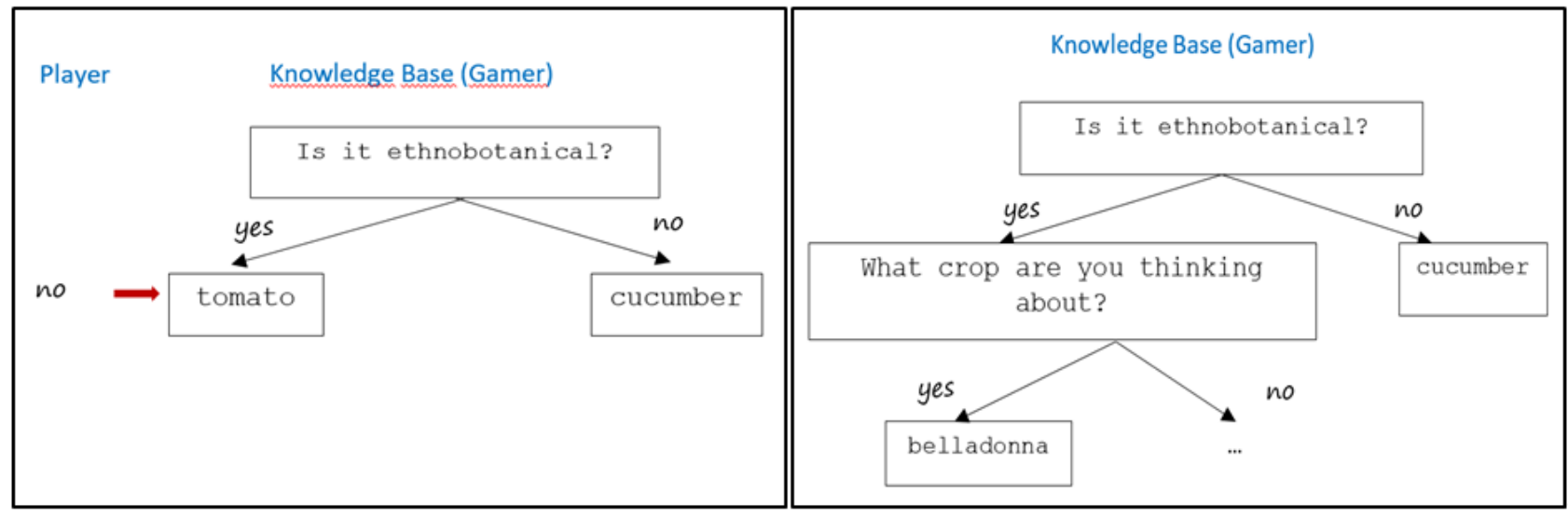

Figure 10. Dynamic construction of the game tree-building a tree (a), adding a subtree (b).

A prototype of the second game has been implemented in the logic programming language Prolog (version SWI-Prolog (SWI Prolog). Although the game is intended primarily for schoolchildren, the prototype was developed (and will be expanded) by university students as course projects in the academic subject of "Introduction to Artificial Intelligence”. Thus, opportunity is also given to teach students mainly in logic programming, presentation of knowledge, and work with recursive structures and algorithms. In order to use the system, a relatively simple user interface has been implemented.

\section{Conclusion and Future Work}

The article presents a distributed environment for game-based learning. The field of application is called an integrated domain because the environment is designed to serve two educational domains - the first one in high school and the other one at university. The use of the environment is demonstrated by two examples.

The first example is used directly in high school. The training of students in a safe environment in STEAM centers 
provides an opportunity to apply didactic technologies and approaches related to the use of virtual and augmented reality, as well as experiment with models close to the real ones. In the conditions of a smart city, the game can be connected with the real physical environment (e.g., the traffic light automation). The use of integrated domains in game-based learning ensures a high degree of activity and motivation of students.

Currently, the second game is realized as a prototype of SWI-Prolog by university students. The prototype will be further improved; our goal is to use the game for teaching in the integrated domain —on the one hand, for training students implementing the game, and on the other hand, for teaching students playing with it. To provide an opportunity to use the game in high school, the user interface will be migrated to the front-end of the distributed environment. The editor discussed in this article will be used to create a new database with building blocks typical of the domain of smart agriculture (for instance, crops). This will also increase the attractiveness of and therefore the desire to use the game. The game itself remains part of the game library, located in the back-end of the environment. The improvement of the game as an expert system of Prologue will continue, mainly for the inclusion of knowledge about new crops in the knowledge base. It is not necessary to reprogram the game for use by students through the new user interface because the built-in two-way interface in SWI-Prolog to the Java programming language will be used.

In the current version of the infrastructure, a personal assistant of a student is implemented. Another future extension of the environment is the development of a personal assistant for a teacher. New games are also going to be designed.

\section{Acknowledgements}

This work is supported by the Bulgarian Ministry of Education and Science under the National Research Program “Smart crop pooduction” approved by Decision of the Ministry Council №866/26.11.2020.

We acknowledge the provided access to the e-infrastructure of the Centre for Advanced Computing and Data Processing, with the financial support by the Grant No BG05M2OP001-1.001-0003, financed by the Science and Education for Smart Growth Operational Program (2014-2020) and co-financed by the European Union through the European structural and Investment funds.

\section{References}

Bahrini, M., et al. (2020). Good vs. Evil: Investigating the Effect of Game Premise in a Smart Home Security Educational Game. In: Extended Abstracts of the 2020 Annual Symposium on Computer-Human Interaction in Play (CHI PLAY '20 EA), Virtual Event, Canada. ACM, New York, NY, USA, pp. 182-187.

Borecki, L., Tolstych, K., and Pok, M. (2013). Computer Games and Fine Motor Skills. Advances in Experimental Medicine and Biology, 755: 343-8.

Bulgarian sectoral strategy for road safety (2021-2030). https://www.mon.bg/bg/143\#Strategy_BDP, assessed on 17.10.2021.

Dobrowolski, P., Hanusz, K., and Sobczyk B. (2015). Cognitive enhancement in video game players: The role of video game genre. Computers in Human Behavior, Vol. 44, 59-63.

Galeote, D. F. and Hamari, J. (2021). Game-based Climate Change Engagement: Analyzing the Potential of Entertainment and Serious Games. Proceedings of ACM Human-Computer Interaction, Vol. 5, No. Chi Play, Art. 226.

Glushkova, T., Stoyanova-Doycheva, A., Ivanova, V., Stoyanov, S., and Doychev, E. (2019). Cyber-Physical-Social Systems and Applications. Part II: Applications, LAP LAMBERT Academic Publishing.

Joshi, A. (2020). Optional Learning in Games, In: Extended Abstracts of the 2020 Annual Symposium on Computer-Human Interaction in Play (CHI PLAY '20 EA), Virtual Event, Canada. ACM, New York, NY, USA, 24-26.

Kahila, J., et al. (2020). Escape Room Game for CT Learning Activities in the Primary School. Proceedings of Koli Calling International Conference on Computing Education Research, Koli Calling’20, Koli, Finland, Article No.: 9, 1-5.

Nistor, G. and Iacob, A. (2018). The Advantages of Gamification and Game-Based Learning and their Benefits in the Development of Education. eLearning \& Software for Education, Vol. 1, 308-312.

Petrov, Epitropova, A., and Stoyanov, S. (2020). Intelligent software agents in help of learning support for autistic students, EDULEARN20 Proceedings, 4622-4628.

Republic of Bulgaria, Ministry of Education and Science. Educational programs, https://www.mon.bg/bg/1690, assessed on 24.10.2021.

Stoyanov, S., Glushkova, Doychev, E., Stoyanova-Doycheva, A., and Ivanova, V. (2019). Cyber-Physical-Social Systems and Applications. Part I: Reference Architecture. LAP LAMBERT Academic Publishing. 
Stoyanov, S., Orozova, D., and Popchev, I. (2018). Internet of things water monitoring for a smart seaside city. XX-th International Symposium on Electrical Apparatus and Technologies SIELA 2018, Bourgas, Bulgaria.

Su Simon, et al. (2021). A Game-Based Approach for Teaching Algorithms and Data Structures using Visualizations. SIGCSE '21, Virtual Event, USA, 1128-1134.

SWI Prolog, https://www.swi-prolog.org/, assessed on 24.10.2021.

Voulgari, et al. (2021). Learn to Machine Learn: Designing a Game Based Approach for Teaching Machine Learning to Primary and Secondary Education Students. ACM Interaction Design and Children Conference (IDC 2021) Athens, Greece, 593-598.

Wang, F.-Y. (2010). The Emergence of Intelligent Enterprises: From CPS to CPSS. IEEE Intelligent Systems, 85-88.

Wooldridge, M. (2009). An Introduction to MultiAgent Systems. Wiley. 